Wo Gegner und Befürworter der Selbstdispensation seit Jahren Gegenteiliges über deren Auswirkungen auf Arzneimittelkonsum und -kosten behaupten, erschien es erfreulich, dass das Bundesamt für Gesundheit BAG 2013 eine wissenschaftliche Studie von neutraler Seite in Auftrag gab. Das Untersuchungsergebnis - der Medikamentenbezug beim Arzt ist nicht teurer als beim Apotheker, sondern eher kostengünstiger - überrascht die Ärzteschaft nicht. Der Umstand aber, dass dieses Ergebnis durch das BAG über die entscheidenden Phasen der Revision des Heilmittelgesetzes hinweg mehr als ein Jahr zurückgehalten wurde, zeigt, dass die Publikation neutraler Studien nicht immer im politischen Interesse der Auftraggeber zu sein scheint.

Dr. med. Jürg Schlup, Präsident der FMH

\title{
Selbstdispensation sorgt für tiefere Medikamentenkosten!
}

\section{Sven Bradke}

Dr. rer. publ. HSG, Geschäftsführer der Ärzte mit Patientenapotheke (APA)

\author{
Nach mehr als einem Jahr veröffentlichte das Bundesamt für Gesundheit (BAG) \\ endlich die erwartete Studie über die Kosten der Selbstdispensation (SD) von Arz- \\ neimitteln. Sie bestätigt aufs Neue, dass die ärztliche Medikamentenabgabe nicht \\ nur geschätzt wird, sondern auch günstiger ist.
}

In 17 von 19 Deutschschweizer Kantonen ist die Abgabe von Medikamenten durch den behandelnden Arzt zulässig. In verschiedenen Kantonen musste dieses Recht der ärztlichen Medikamentenabgabe mit politischen Mitteln verteidigt oder erkämpft werden. Erinnert sei beispielsweise an die drei Zürcher Abstimmungen sowie an den Volksentscheid der Schaffhauser Stimmbürgerinnen und -bürger.

\section{Unabhängige Studie}

$\mathrm{Zu}$ den Kosten der unterschiedlichen Abgabekanäle wurden in den vergangenen Jahrzehnten zahlreiche

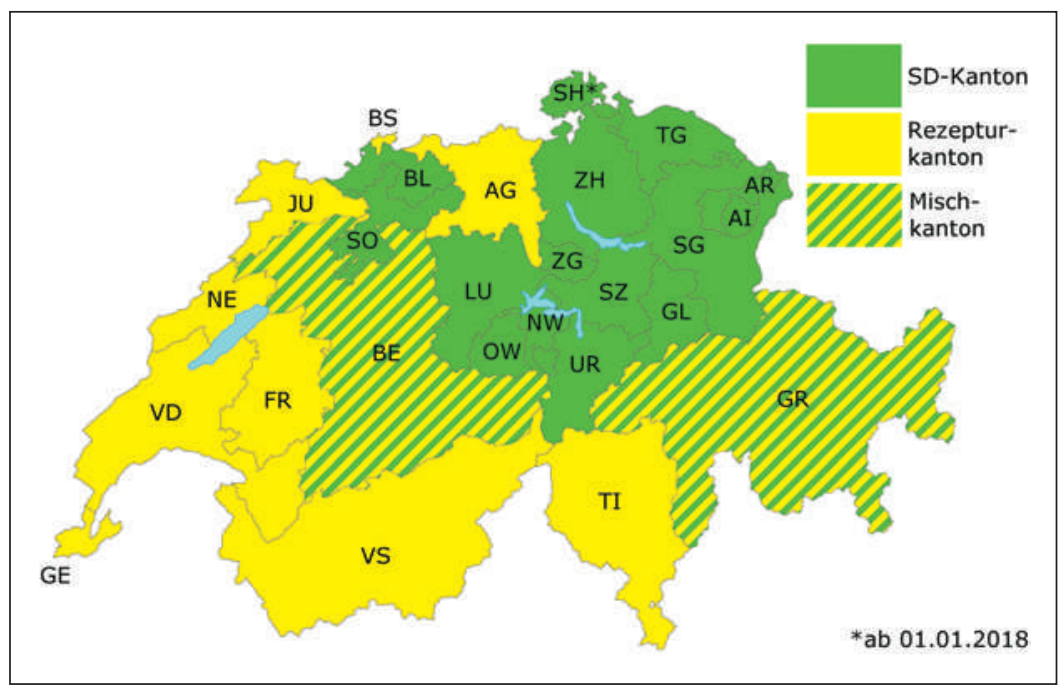

In 17 von 19 Deutschschweizer Kantonen ist die Selbstdispensation zulässig und politisch fest verankert.
Untersuchungen durchgeführt. Je nach Studie und Herkunft tauchten jeweils sehr unterschiedliche Kritikpunkte auf. Aus diesem Grund gab das Bundesamt für Gesundheit (BAG) im Jahre 2013 beim Institut Polynomics eine unabhängige Studie über die Auswirkungen der Medikamentenabgabe durch die Ärzteschaft (Selbstdispensation) auf den Arzneimittelkonsum und die Kosten zu Lasten der OKP in Auftrag. Ziel war es, die Auswirkungen der Selbstdispensation (SD) auf die Medikamentenwahl sowie auf die Kosten zu Lasten der obligatorischen Krankenkasse zu untersuchen. Die aufgrund ihres Forschungsdesigns ansprechende Studie bestätigt, was die Ärzteschaft schon seit jeher verlautbarte: Die ärztliche Medikamentenabgabe ist der günstigere Abgabekanal als die Rezeptur. So, wie dies die jährlichen Statistiken der santésuisse zu den Medikamentenkosten pro versicherte Person und Kanton immer schon zeigten.

\section{Datensätze von Helsana-Versicherten}

Die breit angelegte Polynomics-Studie vergleicht die effektiven Medikamentenkosten von SD-Patienten mit solchen, die ihre Arzneimittel mit einem Rezept in einer Apotheke beziehen. Die Analyse basiert auf zwei verschiedenen Datensätzen der Helsana-Gruppe. Der eine Satz vergleicht Zahlen des klassischen SD-Kantons Luzern mit jenen des Rezeptur-Kantons Aargau. Der andere Datensatz umfasst Versicherte aus den Kantonen Freiburg (SD nur im Ausnahmefall), Thurgau (SD die Regel) sowie Schaffhausen, Zürich und Bern (alle- 
samt gemischte Systeme). Insgesamt stützt sich die Studie somit auf rund 338000 Datensätze.

\section{Medikamente vom Arzt sind 13\% günstiger}

Der Vergleich der Medikamentenkosten zwischen dem SD- und dem Apotheken-Kanal liefert insbesondere unter Berücksichtigung der Leistungsorientierten $\mathrm{Ab}$ geltung (LoA) ein eindeutiges Ergebnis. Er zeigt, dass SD-Patienten rund 13\% niedrigere Medikamentenausgaben haben als solche, die ihre Arzneimittel in Apotheken beziehen. Ohne LoA liegen die Kosten des SDKanals immer noch 6\% unter dem Apotheken-Kanal. «In beiden Schätzungen ist der Einfluss der SD statistisch signifikant und negativ» [1]. Darüber hinaus belegt die Studie einmal mehr, dass SD-Ärzte häufiger Generika verschreiben. "Auch die Wahrscheinlichkeit, dass eine besonders preisgünstige Alternative gewählt wird, ist bei SD-Patienten höher» [2]. Bei bestimmten Wirkstoffgruppen, namentlich Säureblockern, verabreichen Ärzte mit eigener Praxisapotheke zudem geringere Dosierungen als ihre Kolleginnen und Kollegen, die keine Medikamente abgeben.

\section{Leicht höhere Behandlungskosten?}

Die Untersuchung brachte allerdings auch zum Vorschein, dass SD-Patienten im Mittel rund 8\% höhere Ausgaben für ärztliche Leistungen aufweisen. Woran das liegen mag, kann die Studie nicht erklären. Denkbar wären Dauerrezepte, die verschreibende Ärzte verabreichen, oder eine umfassendere Betreuung von SD-Patienten. Weitere Erklärungen könnten auch eine bessere Compliance oder eine spätere Überweisung in Spitäler sein. Die Vereinigung Ärzte mit Patientenapotheke (APA) wird dieser Fragestellung eine verstärkte Aufmerksamkeit widmen. $\mathrm{Zu}$ beachten gilt zudem auch, dass die SD-Kantone in der Regel tiefere Tax-

\section{Die wichtigsten Resultate im Überblick}

- Die Medikamentenausgaben sind bei SD-Patienten unter Berücksichtigung der LoA rund $13 \%$ niedriger. Exklusive LoA sind die Ausgaben immer noch ca. $6 \%$ tiefer.

Korrespondenz:

Dr. Sven Bradke

Ärzte mit Patienten-

apotheke (APA)

Postfach 148

CH-9008 St.Gallen

Tel. 0712465140

www.patientenapotheke.ch ten höher.

- Die Ausgaben für ärztliche Leistungen sind bei SD-Patienten rund $8 \%$ höher. Die Patienten weisen scheinbar auch mehr Konsultationen auf.

Die Studie kann unter www.bag.admin.ch eingesehen werden. punktwerte als die Nicht-SD-Kantone aufweisen. Folglich dürften sich leicht höhere ärztliche Leistungen so wieder ausgleichen.

\section{Zufriedenheit und Qualität nicht beurteilt}

Die Studie kann bedauerlicherweise keine Aussagen über die Qualität der medizinischen Behandlung oder die Zufriedenheit der Patienten machen - die Ergebnisse sind somit rein quantitativer Natur. Es darf aber angenommen werden, dass im Falle der ärztlichen Medikamentenabgabe eine bessere Compliance erzielt wird. SD-Ärzte verfügen nicht nur über eine umfassende Krankengeschichte ihrer Patientinnen und $\mathrm{Pa}$ tienten, sondern sie kennen auch die möglichen Originalpräparate und Generika, deren Wirkungen und Nebenwirkungen sowie allfällige Interaktionen aus ihrer täglichen Arbeit. Letztlich wissen wir auch, dass die Patientinnen und Patienten es ausserordentlich schätzen, ihre Medikamente direkt in der Praxis zu erhalten. Das haben verschiedenste Umfragen immer wieder gezeigt.

\section{Veröffentlichung verzögert?}

Obwohl der Schlussbericht der Studie als Datum den 10. Februar 2014 trägt, veröffentlichte das BAG sie erst Ende Mai 2015. Es darf vermutet werden, dass das Departement von Bundesrat Alain Berset die Publikation der Ergebnisse aus taktischen Gründen verzögerte zumindest solange, bis die Eckwerte der Revision des Heilmittelgesetzes (HMG) in den beiden Räten beschlossen waren. Denn die Verwaltung hatte sich wohl andere Ergebnisse erhofft, sie wollte die Selbstdispensation früher sogar einmal verbieten oder zumindest einschränken. In Erinnerung bleibt beispielsweise der mediale Auftritt von Bundesrat Couchepin, der die Selbstdispensation als den teureren Abgabekanal bezeichnete. Das Gegenteil ist der Fall, wie die Studie einmal mehr belegt. Wir danken dem BAG für die Erteilung des Studienauftrags. Eine zeitgerechtere Veröffentlichung wäre angesichts der HMG-Revision sicherlich wünschenswert gewesen. Die Resultate bestätigen nun aber offiziell, was wir seitens der Ärzteschaft immer schon kommuniziert haben.
1 Polynomics-Studie, Dr. Maria Trottmann et al., Auswirkungen der Medikamentenabgabe durch die Ärzteschaft (Selbstdispensation) auf den Arzneimittelkonsum und die Kosten zu Lasten der OKP, S. 58. 2 ebd., S. 58. 